\title{
Manchester United, capitalismo global y resistencia local ${ }^{1}$
}

\author{
Chris Porter \\ gustavolopez-3@hotmail.com
}

\section{Cómo citar este artículo:}

Porter, C. (2019). Manchester United, capitalismo global y resistencia local (Traductora Jaimes-Peñaloza, S.). TransPasando Fronteras, (13). doi: 10.18046/retf.i13.3492

${ }^{1}$ Nota del editor: traducción de Sonia M. Jaimes. Título original "Manchester United, global capitalism and local resistance", en, Chris Porter, « Manchester United, global capitalism and local resistance », Belgeo [Online], 2 | 2008, Online since 20 October 2013, connection on 02 October 2016. URL: http://belgeo.revues.org/10271; DOI: 10.4000/belgeo.1027.

Agradecemos al colega Chris Porter por conceder el permiso para la traducción y publicación de este artículo- Asimismo a la revista Belgeo. Revue belge de géographie por su consentimiento. 


\section{Resumen}

La adquisición en 2005 del Manchester United por el empresario norteamericano Malcom Glazer- así como la oleada de acciones seguidas por otros clubes de futbol inglés- sucitó muchas preocupaciones entre la hinchada, notablemente en cuando al rol jugado por los clubes en el seno de sus "comunidades".

Es así como las nociones de propiedad han sido confrontadas tanto como las de responsabilidad alrededor de este tipo de "activos" locales son cada vez más pasados por la lupa, no solo por los responsables encargados de reglamentar y de dirigir la industria del fútbol, sino también por los gobiernos a escala local y nacional, los medios y por quienes reivindican implicaciones más grandes, y sin duda mucho más apasionadas: la de los hinchas.

Este artículo presenta algunas de las consecuencias culturales más importantes para los hinchas del Manchester United relacionadas con este asunto. Describe la movilización de la hinchada que se opuso a la compra de Glazer, tanto como las "caídas" culturales de los hinchas. Este caso, supera las nociones simplistas tanto como las confrontaciones banales entre lo "local" y los "global". Pone en evidencia las implicaciones culturales y políticas eventuales seguidas por la inclusión continua del capitalismo global en la vida cotidiana de las personas. 


\section{Manchester United, global capitalism and local resistance}

\section{Abstract}

The 2005 acquisition of Manchester United by American businessman Malcolm Glazer - along with subsequent takeovers of other English football clubs - has raised many concerns amongst supporters, with questions increasingly being asked of the roles football clubs play within their "communities".

Contested notions of ownership and responsibility around such local "assets" are therefore under increased scrutiny, not just from those charged with regulating and governing the football industry, but from local and national government, the media and those who perhaps claim the biggest, and certainly the most emotional stakeholding of all - the supporters.

This paper outlines some of the most pressing cultural implications this issue has raised for Manchester United fans. Accordingly, the mobilisation of supporters opposed to the Glazer takeover is documented, along with the resulting cultural "fall out" for fans. Going beyond simplistic notions of a clash merely between "the local' and "the global", this case highlights some of the cultural and political implications that global capitalism's continuing encroachment into people's everyday lives can have. 


\section{Contexto de investigación y notas metodológicas}

Este artículo es un borrador de la información reunida como parte de una investigación en curso concentrada en la cultura futbolística de Manchester; específicamente apunta a registrar las implicaciones de recientes transformaciones que han impactado en los hinchas de los clubes de fútbol de la ciudad.

La información empírica individual y los grupos focales han complementado la investigación etnográfica acerca de la cultura futbolística de Manchester. De este modo, ha sido posible desarrollar - tal vez de manera privilegiada y ciertamente "comprometida" - una perspectiva analítica desde la que se consideran asuntos sin resolver estructuralmente como lo son los "hábitos" de la cultura futbolística de Manchester.

Como investigador, las implicaciones de este tipo de metodologías de observación participativa son algo bien conocido, y en efecto la naturaleza de "inmersión" del proyecto ha sido asunto de reflexiones significativas de principio a fin. El alcance y rango de este artículo restringe el análisis que se puede presentar acerca de los asuntos estudiados, más bien remite a ofrecer una breve justificación metodológica.

El proyecto de investigación está centrado fundamentalmente en aspectos culturales, en los significados, valores, acciones y discursos, aunque en un entorno influenciado por muchos otros factores estructurales que podrían ser objetivamente tratados desde una perspectiva más individual. Por lo tanto, una clave objetiva de la investigación ha buscado proporcionar un enfoque hermenéutico de las respuestas culturales acerca de transformaciones más amplias. Siguiendo los preceptos de Gadamer e incluso los de Rorty, Foucault y Bauman, esto para documentar en un sentido signifi- 
cativo las implicaciones existentes para aquellos que viven dentro de la cultura local, es necesario experimentar con ellos desde una perspectiva anclada (Blackshaw, 2005). Simultáneamente se produce una transición del participante al investigador y viceversa, circunstancia que ciertamente genera problemas que requieren reflexiones explícitas, los puntos de vista culturales y naturalmente los que están incluidos en este tipo de posturas deben ser vistos tanto como un fuerte argumento justificador cuando se elaboran las criticas metodológicas del caso.

\section{El contexto}

En mayo del 2005 el empresario norteamericano Malcom Glazer se convirtió en el accionista mayoritario del Manchester United Plc, muy a pesar de la opinión de muchos de los hinchas y accionistas del club. En solo dos meses compró más del 98\% de las acciones de la compañía, y como nuevo dueño del club rápidamente lo retiró del mercado de valores, forzando con ello una compra obligatoria de los remanentes de los accionistas "disidentes".

El Manchester United había estado en el mercado accionario en 1991, y había existido como un club de fútbol de propiedad privada desde su formación en 1878. Muchas preguntas se hicieron públicamente en esos 14 años a la empresa, particularmente a lo que se vio como un conflicto inherente entre los intereses de quienes ven al club de fútbol como algo de interés común, y quienes lo ven públicamente como una empresa con obligaciones de producir beneficios y dividendos para los accionistas (Conn, 1997; Brown y Walsh, 1999). Los accionistas comunitarios -los hinchas del club- nunca tuvieron nada más que apuestas emocionales en el club hasta que la oportunidad de comprar se presentó 
de manera tangible en el club. Sin embargo, asegurar algo más que una participación accionaria, probó más allá del alcance financiero de la mayoría de los hinchas, quienes en conjunto no vieron sus acciones más que en recuerdos, certificados y oportunidades de asistir a la empresa AGMs.

En octubre de 1998, la radiodifusión satelital del barón de los medios Rupert Murdoch de la compañía BSkyB lanzó la oferta de adquisición por el club que fue eventualmente bloqueada por los Monopolios y la Comisión Mergers, tras la presión de la bien organizada campaña de los hinchas independientes quienes hicieron lobby al MPs a fin de desafiar al movimiento por motivos de competencia injusta (ver Brown y Walsh, 1999).

Los hinchas más politizados del Manchester United, principalmente por la vía de la asociación de hinchas independientes del Manchester United (IMUSA) ${ }^{2}$ de manera independiente produjeron los fanzines Red Issue y United We Stand, para oponerse vehementemente a cualquier adquisición del club. Ellos veían constantemente su status de PLC como algo lejano y parte de una perfecta situación, ellos veían con temor las motivaciones de todo aquel que buscaba llevar al club a manos privadas y alejarlo de la responsabilidad pública.

Siguiendo el miedo de la oferta de Murdoch, los hinchas se vieron urgidos de comprar acciones del club y confiárselas a los Shareholders United ${ }^{3}$-una organización conformada por hinchas

2 IMUSA fue formada en 1995 como una campaña independiente, reúne un amplio espectro de problemáticas relacionadas con los apoyos de las hinchadas por aquel entonces. ${ }^{3}$ Su aumentó sus intentos por movilizar las acciones de valores de los hinchas en el periodo en que Murdoch intentó realizar la posesión del club en 1998, bajo el nombre original Shareholders United Against Murdoch (SUAM). 
con el objetivo de reunir la participación de los hinchas propietarios y darles el poder necesario para bloquear cualquier intento futuro de adquisición, y con la ambición de estar en una posición de darle control accionario a los hinchas, convirtiendo de este modo al Manchester United en un club de fútbol democrático constituido por sus hinchas como propietarios.

A pesar del alto valor alcanzado en el mercado por la compañía, y pese a que muchos hinchas se unieron al club como accionistas (había alrededor de 32.000 accionistas individuales en el momento en que Glazer hizo su postulación de compra accionaria), los hinchas asociados no estaban en condiciones de poder prevenir la compra de Glazer. Aunque hubo un alto número de demostraciones de alto perfil que desde octubre de 2004 cuando la opción de compra se volvió un hecho altamente probable, mantuvieron el tema en los medios de comunicación otorgándole apoyo generalizado a la hinchada del club, aún así ello no impidió que Glazer ejecutara su compra.

Hubo protestas y marchas afuera del Old Trafford, en los días de partido se exhibieron pancartas, trapos y se cantaron consignas que expresaban que los hinchas no permitirían la venta del club. "United, United no está a la venta" fue el estribillo general, junto a las pancartas, trapos y folletos en los que se leía el mensaje "Sin clientes no hay beneficios", se instaba a los hinchas a efectuar un boicot a la idea del club como mercancía. Una efigie de Malcom Glazer quemada afuera del Old Trafford no les dejó duda a los observadores el tipo de bienvenida que le esperaba al nuevo dueño del equipo. Lejos del estadio hubo protestas, la hinchada rodeó, instigó y presionó las tiendas de Nike, Vodafone y Ladbrokes pertenecientes a los patrocinadores del equipo y ubicadas en el centro de la ciudad, irrumpieron en una reunión de los ejecutivos de la UEFA en la ciudad de Manchester para cuestionar la nueva 


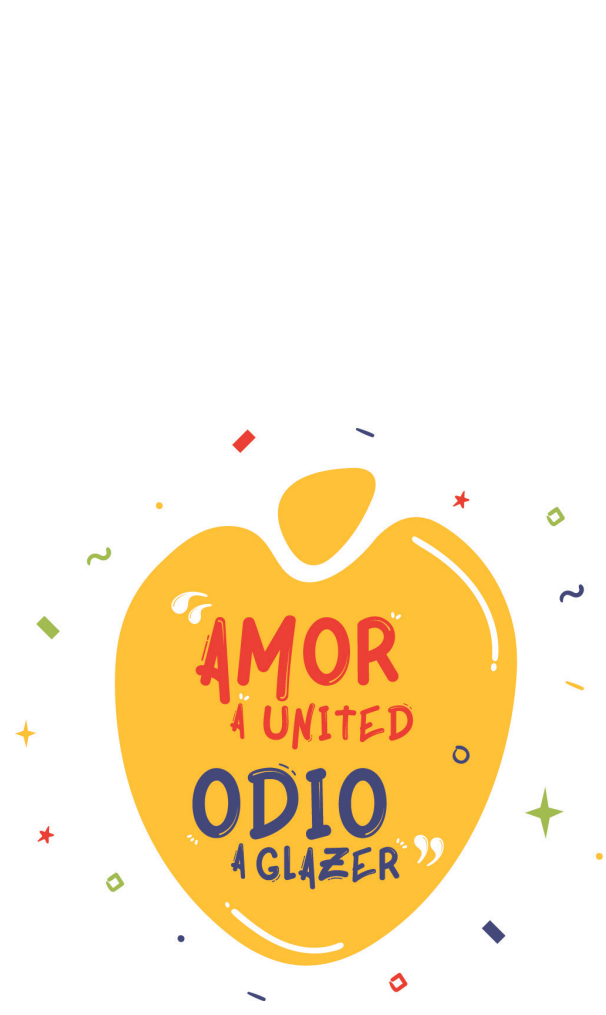

\section{"United, United no está a la venta"}

\section{"Sin clientes no hay beneficios"}

organización administrativa, y Manchester seguía adornada con calcomanías con el slogan: "Amor a United, odio a Glazer", frase que se ha convertido en omnipresente del discurso de la hinchada.

Aunque al parecer la mayoría de los hinchas del Manchester United han estado en la oposición, una vez se posesionó el nuevo dueño muchos no vieron el sentido de seguir protestando contra los nuevos dueños del club, y el punto de vista que primó fue el de conservar el tradicional lugar de una hinchada que apoya a su equipo de futbol sin importar el tipo de dueño que se tenga.

El estatus de propiedad privada ha sido, después de todo, el estado que el club había tenido durante gran parte de su historia, y ciertamente a partir de los años sesenta del siglo XX los dueños del club habían cortejado con regularidad la controversia y habían estado lejos de obtener el apoyo popular de los hinchas del United (véase Crick, 1989; Conn, 1997). 
De la misma manera, los hinchas de Gran Bretaña nunca habían tenido una representación democrática clave en lo relacionado con los procesos de decisión, de modo que, podría decirse que se había desarrollado una especie de cultura de dependencia fatalista hacia sus equipos, que simultáneamente empoderaba a los clubes a explotar y disfrutar de las "audiencias cautivas" (Conn, 1997). Sin embargo, la experiencia de los años recientes produjo formas más politizadas y frecuentemente con elementos más militantes ente la hinchada del Manchester United, muchos de esos hinchas no tenían intención de continuar en ese rol de servilismo bajo lo que ellos denominaron la ocupación de su club por parte de Glazer.

\section{Fútbol inglés y capitalismo global}

Como parte de una industria, e incluso de una nación, cuya estructura e intereses están explícitamente relacionados (podría decirse que son motores de dicha relación) las dinámicas de flujo de capital el club de fútbol Manchester United ha llegado a ser considerado una mercancía, es decir, empezó a ser visto como un commodity, y ha empezado a ser tratado de ese modo. Dicho commodity se ha construido desde una idea relativamente borrosa de comunidad o de cultura, y ambas, a su vez, están direccionadas y construidas en vías opuestas, desde las lógicas de una ideología capitalista global muy "dura", que parece muy difícil de manejar para quienes ven al Manchester United como un fenómeno con el que se puede aumentar su fortuna.

Dentro de esta globalización, el entorno corporativo y mercantilizado, así como las nociones de identidad local, capital cultural y autenticidad se han convertido cada vez más en objeto de escrutinio en el discurso de los hinchas del Manchester United, 
quizás no sorprenda en este momento, cuando estos valores son vistos como los más amenazados. Los cuestionamientos a la identidad de los hinchas, así como a su credibilidad no es algo que sólo haya ocurrido en la coyuntura en la que la saga Glazer se posesionó en el club. La popularidad del fútbol inglés aumentó y refundó una nueva credibilidad dentro de un espectro social mucho más amplio durante los años noventa del siglo XX lo que interrumpió las nociones tradicionales de cultura futbolística inglesa, e indudablemente puso las bases sobre las cuales el juego se convirtió en una industria digna de atención a los ojos de inversionistas como Malcom Glazer.

De hecho, fue este creciente valor del fútbol inglés como producto lo que convenció a Rupert Murdoch para realizar en última instancia su apuesta por el Manchester United en 1998 un equipo que por entonces estaba condenado al fracaso. Comprender este episodio de la reciente historia del club es crucial para entender la cultura de su hinchada, tanto la que existe desde entonces como la actual, así como para comprender mejor las reacciones que hubo entre los hinchas del Manchester United en el 2005 ante la posesión de Glazer.

\section{Identidad local}

Tradicionalmente, los clubes de fútbol han estado vinculados tanto a espacios físicos como a sitios simbólicos, una evidencia de ello son sus nombres los que en pocas ocasiones adoptan los nombres de las ciudades o pueblos donde se crean y funcionan. Aunque existen estudios en los que se muestra que algunos clubes de fútbol han conseguido hinchas de fuera de sus lugares de procedencia, frecuentemente esto sucedió en las primeras décadas del siglo 
XX (Mellor, 1999), ciertamente en el Reino Unido existen pequeñas dudas acerca del ambiente contemporáneo que se vive en el fútbol, y especialmente en la cima del nivel del juego inglés, lo que ha sido tema de debate en lo referente al rol de los clubes de fútbol como símbolos representativos de identidades locales tradicionales. Esto ha sido especialmente exacerbado a la luz de varios clubes que han aumentado sus aspiraciones de globalización (Brown, 1998; King, 1998; Giulianotti, 2002; Crabble and Brown, 2003), lo que ha empujado al fútbol inglés a estar al frente del proceso de mercantilización de este deporte desde la primera etapa de la década del noventa del siglo XX (Conn, 1997). Durante este tiempo, el fútbol ha emergido como el deporte más globalizado, particularmente por el tipo de relaciones que ha establecido con los medios y las tecnologías de comunicación globales.

Estas tendencias globalizadoras han creado nuevas audiencias local, nacional y globalmente. Esto inclusive ha causado dislocaciones de lo que era "tradicional" (i.e., desde hace mucho tiempo y/o de manera local) en cuanto al el sentido de identidad propia de los hinchas con relación a sus clubes de fútbol (Brown, 1998; King, 1998; Brown y Walsh, 1999), un modelo identificado por la taxonomía elaborada por Giulianotti (2002) para identificar las identidades de los "tipos" de hinchas. Entre estas formas tradicionales y nuevas de consumir el fútbol, las nociones de localidad y de ciudad, se juega el lugar de la cultura popular.

Frecuentemente, las nociones de autenticidad -que en el caso de Manchester se vincula fuertemente con un sentido de pertenencia a la ciudad- están muy mediadas por los medios y la mercantilización cultural, de modo que son elevados y potencialmente cuestionados de amplias y múltiples maneras las nociones de globalización, sus características y sus consecuencias. 


\section{El contexto futbolístico de Manchester}

Una manifestación particularmente clara en este contexto es la concerniente, evidentemente, compleja y frecuentemente incompatible relación entre las imágenes públicas y las políticas corporativas de los dos principales clubes de fútbol de Manchester. Unidos son reconocidos como los líderes en este campo y el club es abiertamente vendido como una marca global. El Manchester City ha empezado a ser mucho más una mercancía de operaciones globales - parcialmente evidenciado por el movimiento que se ha realizado desde su tradicional calle Maine, conocida como su "casa" a una nueva sed, mucho más benéfica y compleja financieramente - así como sucedió en el 2007 con la toma de posesión de la firma tailandesa Thaksin Shinawatra PM. Simultáneamente, City continúa explicitándose como una marca del club "local" y tradicional de Manchester.

Vale la pena enfatizar de nuevo que la hinchada del Manchester United había percibido su identidad local y su cultura tradicional bajo amenaza mucho antes de que Glazer tomara posesión de su cargo. Como ya se mencionó, durante los años noventa del siglo XX hubo transformaciones en el fútbol inglés que, en forma extrema, lo comercializaron; acciones que fueron parte esencial del Manchester United y que condujeron a fuertes críticas y debates hacia el club incluso desde su propia hinchada. Mientras se estaba disfrutando del periodo más exitoso en la historia del club, se produjo un cisma imaginario en las mentes de muchos de los hinchas del United y entre el equipo y quienes dirigían y controlaban el club, por lo tanto, la frase usada entre los hinchas de cultura independiente "ama al equipo-odia al club" estuvo muy bien empleada (King 1998; Brown 2004). Como el más apoyado, el más conocido, y durante este tiempo el más exitoso club en térmi- 
nos deportivos y comerciales, Manchester United se auto fundó como el centro de la mayoría de los debates relacionados con el estado del fútbol en Inglaterra.

Como hinchas del club, que era tan amado como odiado, estos aficionados se convirtieron en blanco de las burlas de las hinchadas rivales y de algunas de las secciones de los medios de comunicación. Muchas de las burlas, que frecuentemente eran de desprecio, se centraron en el sentimiento que sostenía que los hinchas del Manchester United carecían de autenticidad, su credibilidad como hinchas leales del fútbol empezó a ser cuestionada, especialmente en lo relacionado con las nociones de localidad. Los hinchas del Manchester United que no vivían en Manchester fueron objeto de burlas, y el estereotipo más común empezó a sostener que los hinchas del Manchester United eran de cualquier parte que no fuera Manchester, la idea se difundió y aceptó sin inconvenientes en el mundo cultural futbolero y más allá de él.

En la cultura inmediata del Manchester, el enfoque sobre lo local fue muy intenso, los hinchas rivales del Manchester City regularmente reclamaban la "propiedad" y "pertenencia" a la ciudad. Cánticos como: "vienes de Manchester?" fue lo que regularmente se escuchó en las voces de los hinchas de la ciudad en los partidos del campeonato entre los dos clubes (este canto se convirtió en la muestra de la mayoría de los rivales en los partidos contra el Manchester United). También se efectuaron burlas referidas al amplio apoyo que se le daba al United en el extranjero. Por ejemplo, los aficionados del City se burlan cantando "eres el orgullo de Singapur", y ello se volvió un tema regular en el discurso de la hinchada, en las canciones que se cantaban en el estadio, en las bromas de los bares hasta en los artículo y cartas de los fanzines, tanto como en los medios de comunicación más convencionales (Crabbe and Brown, 2003; Brown, 2004). 
Recientemente, las iniciativas de mercadeo oficial del Manchester City han resaltado los reclamos efectuados or parte del club que acreditan su autenticidad, tendencia que se destacó en la campaña del 2006, la que consistía en un despliegue de vallas publicitarias ubicadas alrededor de Manchester donde se leían los slogans: "Esta es nuestra ciudad" y "Manchester Real" contrastados con los colores del club urbano (Burrell, 2005). Como parte de la respuesta de este tipo de "ataques" contra la credibilidad y la autenticidad de la hinchada del club y de los manchesterinos, los hinchas del Manchester United fueron vistos como individuos que buscaban reafirmar y reevaluar su identidad local (King, 1998; Crabbe and Brown, 2003), esto fue así, en parte por la re-intensificación de su rivalidad con el City, lo que fue una reacción descrita por Brown como una variante asociada con el concepto de Appadurai (1996) según el cual "crear una diferencia cultural con el 'otro' " tanto como con el concepto de Robertson (1992) según el cual se trata de trabajar en la resignificación de la "glocalización" (Brown, 2004, p. 8).

\section{Globalización, mercantilización y la disputa por la propiedad cultural}

La globalización del fútbol inglés, y particularmente la del Manchester United, ha tenido claramente un impacto significativo no sólo sobre la comercialización adoptada por el tipo de "producto", también ha incidido en los "consumidores" operando en sus complejas dinámicas de hinchar y en la cultura de apoyar a sus equipos, mucha de la que está basada en nociones de identidad local. Irónicamente, el club, que ha sido visto como un sujeto de vanguardia en la globalización del juego, y cuyos hinchas se han ridiculizado cotidiana y rutinariamente como ejemplo de lo no-local, el rostro de lo no auténtico de la cultura futbolística globalizada es el Club 
donde parece haber existido la oposición más vehemente a este tipo de transformaciones. La feroz campaña de resistencia de los hinchas del Manchester United contra la compra del equipo en 2005, y la resonancia cultural que dejó, no puede simplificarse a una mera batalla entre "lo local" y "lo global". Este caso representa un ejemplo intrigante de las dificultades enfrentadas cuando una forma de cultura local se convierte en un asunto de intereses comerciales globalizados, y de cómo la identidad local se convierte, al mismo tiempo, en un asunto amenazado y revitalizado por la exposición a las fuerzas mundiales.

La oposición contra la compra del Manchester United por parte de su hinchada, y más específicamente la búsqueda de su alma sin precedentes que muchos experimentaron a raíz de esa compra, ha provocado nuevas dinámicas culturales entre los hinchas del club. Este tipo de reflexiones individuales y colectivas, como las describe Giddens (1994) muestran como la globalización puede fomentar la construcción de una mayor conciencia sobre algunos antiguos y subconcientes aspectos de formación identitaria, lo que ha conducido, a cuestionar algunos de los elementos fundamentales de los valores y miradas de los hinchas.

Los debates emocionales han sido desarrollados por las personas y en las imprentas; y han forzado a los hinchas a preguntarse a sí mismos qué es lo que los une cuando expresan sus lealtades al club. Por lo tanto, los hinchas examinan detalladamente lo que sentían hacia el valor relativo que pusieron a todos estos aspectos del "club", incluyendo los dueños, los jugadores, los dirigentes, el estadio, la historia del club, la ciudad, los hinchas, las canciones, los rituales, la camaradería, las rivalidades, las experiencias compartidas y las herencias familiares. Los hinchas han reflexionado sobre el tipo de relación que ellos desean con su club, de modo 
que, para muchos el poder entre los dueños y los hinchas, lo que previamente no había sido un asunto por mencionar como lo era el asunto de los productores y los consumidores, se fue convirtiendo en un problema de primer orden.

Para muchos, la respuesta a este tipo de problemas significó que no se podía seguir poyando al club de la misma forma que se había hecho previamente. Algunos renunciaron a seguir asistiendo a los partidos, mientras que otros decidieron seguir asistiendo, pero evitando financiar al régimen de Glazer hasta donde les fuera posible, restringendo los gastos a la compra de sus entradas, o tal vez sólo asistiendo a los partidos jugados fuera del ámbito local. Para la mayoría, no había que realizar una decisión - sin hablar de los problemas de quienes adquirían y se adueñaban del club, de la deuda y de los accionistas-, los hinchas nuevos continuaron asistiendo al Old Trafford desde la compra de Glazer.

Para quienes habían decidido dejar de asistir a los partidos, el plan inicial para mantenerse visibles y para hacer escuchar sus protestas contra los nuevos dueños fue rápidamente algo discontinuo no sólo por la apatía de muchos hinchas, sino por los inesperados niveles de hostilidad que algunos hinchas imprimieron a esas protestas. Como alternativa, los protestantes debían idear medios menos conflictivos para que sus voces de oposición quedaran registradas, ello a fin de no provocar altercados desagradables entre los hinchas, ni para motivar las divisiones inseparables entre la hinchada del club.

Una dinámica interesante y controversial en la cultura del fútbol local de Manchester fue el resultado de esas divisiones, en la forma del FC United de Manchester - un club configurado por el desencanto y la marginalización de los derechos asociados con la situación del Manchester United-. Formados en la imagen 
del club, los hinchas querían que el Manchester United fuese, un nuevo club dirigido democráticamente, en el que cada miembro tuviera igualdad de voz para sugerir como debía ser su dirección. Su constitución (votada por 4.000 miembros fundadores, tanto como por los miembros de la Junta, el nombre del club y la cúpula) estableció una visión de un club de fútbol fuerte, con lazos orgánicos con la comunidad en el que nunca habría un interés por anteponer las preocupaciones comerciales sobre las de los hinchas que apoyaban al equipo en cada partido.

A partir de las divisiones en grupos de diez de la Liga Premier Inglesa, el FC United clamó por proporcionar una experiencia futbolera regular y accesible para aquellos hinchas del Manchester United que entonces rechazaban, o no podían pagar para asistir a los partidos en el Old Trafford, aunque muchos de los que asistían a los partidos de la FC United seguían hinchando activamente al Manchester United. Para aquellos hinchas que boicoteaban cualquier apoyo al Manchester United, la retórica de "amor al equipo, el odio al club" se ha ido transformando en formas más complejas, de modo que, para muchos, la mirada de "amor a la historia, odio al presente" se ha vuelto más precisa. Para ellos, es de esta manera es como se han moldeado los recuerdos compartidos, los que se han vuelto como bonos con los que se puede ver el espíritu "intangible" o el "alma" del Manchester United, lo que ha legitimado su decisión de romper el hábito cultural de toda la vida.

\section{Bienes culturales y regulación}

El nivel de sentimientos entre los hinchas del Manchester United concerniente a lo que ellos continúan viendo como la amenaza a su identidad cultural, plantea importantes interrogantes 
en términos de cómo las instituciones culturales y las prácticas pueden seguir siendo protegidas. Aunque el sistema legal y político en el que opera la industria del fútbol inglés en gran medida impide las intervenciones en lo que se considera asuntos de índole privada, existen algunas áreas en las que el gobierno o las regulaciones gubernamentales previenen que el fútbol u otros "activos" queden a merced total de la economía de mercado. Temas como los niveles de endeudamiento, la solvencia financiera, el acceso público y los acuerdos televisivos están exentos de regulación en varios de los reglamentos, de hecho el intento de compra del Manchester United por parte de la BSkyB de Rupert Murdoch en 1998, fue bloqueado por los monopolios y fusiones de la Comisión Merger bajo el argumento de la competencia desleal, aunque el hecho fue mucho más revelador sobre lo que estaba sucediendo con la industria televisiva que con el futuro del fútbol (Brown and Walsh, 1999).

Existen, ocasionalmente, acciones realizadas para proteger lo que es visto como un activo de la cultura o el deporte nacional, aunque en el caso de la compra efectuada por Malcom Glazer, no hubo procedimientos regulatorios ni sistemas que impidieran la acción, en últimas, se trató de una transacción perfectamente legal. Y cualquier efecto adverso ocurrido entre las partes involucradas no era parte de las preocupaciones de la Asociación de Fútbol, la Premier League, UEFA, FIFA o el gobierno inglés. De hecho, la única organización que declaró algún interés en el proceso relacionado con la intervención fue la Liga de Fútbol Nacional Americana quien expresó su interés con relación a que los dueños de los Bucaneros de Tampa Bay, la familia Glazer estuviera posiblemente jaqueando la estabilidad financiera de uno de los clubes miembros al comprometer demasiado capital en la nueva aventura empresarial (Griffiths, 2005). 
Existe, hace relativamente poco, un área de regulación en el fútbol inglés, con la que se habría prevenido la oferta pública de compra tanto de Murdoch como de Glazer, y con lo que de hecho se habría impedido la comercialización en bolsa del Manchester United, es la ya desaparecida regla 34 de la FA. Esta regla esencialmente prevenía a los clubes de fútbol ser empleados para hacer dinero por inversionistas, pues limitaba el pago de las utilidades, algo con lo que se preveía como una amenaza potencial para el juego, y al papel que este tenía para la comunidad en términos generales, cuando fue introducida por la Asociación de Fútbol a finales del siglo XIX (Conn, 1997).

Casi por 100 años, los clubes de fútbol fueron regulados para proteger a sus comunidades de los accionistas y de las amenazas de las fuerzas del mercado, de modo que se "garantizaba que los clubes se mantuvieran como instituciones deportivas" (Conn, 1997, p. 41). El club de fútbol Tottenham Hotspur, sin embargo, evadió la regla 34 en 1983 por la reestructuración interna que se produjo e hizo del club de fútbol un simple subsidiario de una compañía filial mucho más grande, la que, por cierto, no estaba técnicamente sujeta a las reglas de la FA. La aquiescencia de la Asociación de Fútbol al permitir el no cumplimiento de esa regla tan fundamental por parte de uno de los clubes de fútbol miembros de la Asociación permitió al Manchester United ser el siguiente en la serie en 1991, cuando como con un Plc fue capaz de pagar altos dividendos a sus directivos después de haber jugado a la bolsa de valores.

La omisión de la regla 34, y más puntualmente la voluntad del gobierno deportivo para permitir que los clubes de fútbol sean tratados como mercancía con una clara preocupación comercial a expensas de los intereses de la comunidad de base que es más cultural, crearon el ambiente en el que Malcom Glazer fue capaz de comprar el Manchester United, y montó al club con una deuda de 
cerca de medio billón de libras, a pesar de la abrumadora oposición de los hinchas del club, tanto como de la fugaz oposición de los directivos y empleados del club. El verdadero miedo de los hinchas desde entonces es que el club, opere sin un esquema de rendición de cuentas pública y que dentro de ese esquema pueda parecer como una industria con regulaciones transitorias y muy flexibles, en la que el objetivo final sea hacer dinero para sus dueños.

Es poco reconfortante que un equipo exitoso como este se vea en la necesidad de ser rentable, particularmente al considerar que los ingresos corrientes más fácilmente disponibles para su explotación son los suministrados por propios hinchas. Para la hinchada del Manchester United, disfrutar de la experiencia diaria de los partidos y apoyar a su equipo siempre ha sido un fin en sí mismo, y sin la regulación que proteja su dominio cultural por largo tiempo, ellos son, hoy por hoy, simplemente las vías mediante las que los propietarios del club seguirán buscando obtener su propio beneficio.

\section{Exposición global, distinción local: ¿de quién es la cultura?}

"Lo local... es una arena donde los significados de los hábitos de varias personas se entrecruzan, y donde lo global, o lo que parece haber sido local en algún lugar, también ha tenido la misma oportunidad de hacerse a sí mismo en casa. En esta intersección, las cosas funcionan bien por si mismas todo el tiempo, entonces el cambio de este año será la continuidad del próximo. Nos preguntamos, entonces de manera simultánea, que es lo que el lugar hace por la gente, y que es lo que la gente hace por el lugar" (Hannerz, 1996, p. 28) 
En la teoría de la globalización, la interacción entre lo local y lo global ha probado ser un tema controversial y con una compleja particularidad, con viejas ideas de flujos de un solo sentido, en el que lo local ha sido concebido como un simple recipiente pasivo en el que caben todo tipo de asuntos, siendo reemplazable por una perspectiva mucho más multifacética en la que lo local es concebido como un agente de la globalización, y por lo tanto no necesariamente como la víctima que con frecuencia previamente se describía (Robertson, 1992; Hannerz, 1996). Las representaciones de "lo local" pueden también caer en la trampa de impregnar el concepto con cualidades ambiguas como tradición y permanencia (Hobsbawm and Ranger, 1983; Anderson, 1991).

El romanticismo por lo local infiere profundidad, o autenticidad, lo que es por contraste ausencia de "superficialidad" global - una inferencia que puede fácilmente proporcionar mala comprensión y simplificación extrema en la mirada. El inconsciente y la naturaleza momentánea de las interacciones entre lo local y lo global debe tenerse en mente al momento de realizar análisis acerca de la vida cultural donde se encuentran los dos bandos, de modo que como infiere Hannerz, no podemos permitir que una cultura local no tenga los elementos que la alimentan.

Es claro que, en la cultura futbolística, la creciente exposición a los flujos globales (Castells, 1996) no han tenido un efecto lineal ni unidimensional en la hinchada del Manchester United, mientras que se tomaba más conciencia de los objetivos globales del club, su alcance y popularidad, las peculiaridades de la cultura futbolística local han permitido reevaluar las identificaciones de los manchesterianos. Al mismo tiempo, ellos eran muy conscientes de la importancia de las dimensiones globales de las negociaciones de su club, dado que esto ampliamente les permitía continuar 
siendo competitivos frente a sus rivales, ambos, local y globalmente. De modo que exhibir la conciencia amplia, o "globalizada" (Robertson, 1992), su identidad local no sólo ha quedado intacta, sino que incluso se podría decir: vigorizada.

Sin embargo, los aspectos prácticos de las operaciones globalmente orientadas al Manchester United, junto con transformaciones más amplias en la industria del fútbol, han incrementado las formas tradicionales y locales de apoyar apreciadas por muchos hinchas que resultaban incongruentes con el nuevo y cambiante entorno, o estructura, en la que están actuando. Si bien podría argumentarse que no existía nada inherente entre la cultura de la hinchada del fútbol local que podía ser rechazada, o quizás mucho más apta de serlo por la exposición a las fuerzas globales, las instituciones y las formas estructurales con las que se les relacionan más allá de las culturas locales -como podría ser la "compañía" del club de fútbol y aquellas organizaciones que dirigen el juego- no han mostrado la capacidad o disposición para combinar adaptación y preservación dentro de este amplio entorno.

Los intentos por gobernar grupos dirigentes y clubes de fútbol, particularmente en la Liga Premier Inglesa, para adaptar el cambio del entorno en el que ellos operan han tendido a favorecer un acercamiento que acople operaciones del club de fútbol a las formas más provechosas de los mercados de los que ahora hacen parte. Por supuesto, esto no puede ser visto como un intento de hacer lo mejor de la situación en la que ellos mismos están, ya que los beneficios percibidos de las fuerzas del mercado han estado explícitamente detrás de muchos de los avances más buscados en el fútbol inglés durante las décadas más recientes (Conn, 1996). Por lo tanto, la estructura del fútbol inglés ha sido moldeada por sus guardianes para sacar ventaja financiera del mercado global, en 
lugar de tener cualquier "valor de mercado" inherente por encima de los competidores más estrictamente regulados.

Con motivos más altruistas, muchos hinchas de fútbol han buscado activamente oponerse a la mercantilización y corporativización de sus clubes de fútbol y del juego en general. El ejemplo particular de los hinchas del Manchester United muestra que mientras el atractivo del éxito local, nacional y global se ha mantenido fuerte las preocupaciones más fundamentales relacionadas con el mantenimiento de una cultura distintiva y local de seguidores ha requerido aumentar la hinchada que se opone con mayor frecuencia a los direccionamientos en los que se esta orientando a su club. La falta de poder de la hinchada en este tipo de preocupaciones ha llevado a muchos a cavar más profundamente, a cuestionar la naturaleza de sus identidades culturales, afiliaciones y relaciones.

Para aquellos hinchas, sostener ideologías fuertemente relacionadas con los derechos de propiedad comunes de una institución local tan importante los ha llevado a considerar el tradicional supuesto según el cual los hinchas deberían tener un papel sumiso en su relación con los clubes de fútbol. Las preocupaciones de los hinchas, ignoradas por mucho tiempo, han empezado a estar más motivadas políticamente, con un resentimiento creciente hacia los encargados de supervisar el desarrollo saludable del juego. Si los guardianes del juego no están dispuestos o son incapaces de proporcionar la tutoría y la directriz moral que los hinchas anhelan, entonces ellos se convertirán más en simples consumidores si ellos desean alterar el curso actual que se está tomando. La democracia de la hinchada es sin lugar a dudas un problema en crecimiento en el futbol inglés, y el ejemplo del FC United representa algo nuevo, en ese sentido el número de hinchas de uno de los clubes de fútbol más "globales" sienten fuertemente que su club 
ha empezado a dislocarse - al menos espiritualmente- de sus raíces locales tradicionales, que se habían hecho lo que previamente se consideraba impensable y formaron su propio club.

Estos hinchas fueron capaces de "salirse" de los roles tradicionalmente definidos y alteraron sus perspectivas en lo que constituye "el club de fútbol" en aras de mantener una lealtad inmaterial a su club, y, de hecho, a fin de re-fundar su apoyo mediante la formación de una "re-ubicación" material para el ahora inaccesible (tanto financiera como éticamente) Manchester United. Este "estado alterado" de identificación ha tenido implicaciones no sólo relacionadas con la perspectiva de la "identidad local", sino también con el compromiso político, en ello los hinchas - a través de su experiencia con las fuerzas del mercado impactando negativamente en sus vidas culturales- han sido también capaces de construir puntos de vista mucho más críticos acerca de los problemas de las cargas políticas y del poder de la propiedad, el consumo, la responsabilidad y la regulación. 


\section{Bibliografía}

ANDERSON B. (1991), Imagined Communities, London, Verso.

APPADURAI A. (1996), Modernity at Large: Cultural Dimensions of Globalization, Minneapolis, University of Minnesota Press.

BLACKSHAW T. (2005), Zygmunt Bauman, Oxon, Routledge.

BROWN A. (ed.) (1998), Fanatics! Power, Identity and fandom in football, London, Routledge.

BROWN A. (2004), "Manchester Is Red? Manchester United, fan identity and the 'Sport City'", in ANDREWS D., Manchester United: A Thematic Study, London, Routledge.

BURRELL I. (2005), "Are you local? City takes on United's 'foreign legion'”, The Independent: Online Edition, http://news.independent.co.uk/media/ article315118.ece, 26/09/2005.

CASTELLS M. (1996), The Rise of the Network Society, Oxford, Blackwell.

CONN D. (1997), The Football Business, Edinburgh, Mainstream.

CRABBE T. and BROWN A (2003), "You're not welcome anymore: The football crowd, class and social exclusion", in WAGG S. (ed.), Football and Social Exclusion, London, Frank Cass.

CRICK M. (1989), Manchester United: the Betrayal of a Legend, London, Pelham.

GIDDENS A. (1994), "Living in a Post-traditional Society", in BECK U., GIDDENS A. AND LASH S. (eds.), Reflexive Modernization: Politics, Tradition and Aesthetics in the Modern Social Order, Cambridge, Polity, pp. 56-108. 
GIULIANOTTI R. (2002), "Supporters, Followers, Fans and Flaneurs: a taxonomy of spectator identities in football", Sport and Social Issues, 26, 1, February 2002, pp. 25-46.

GRIFFITHS K. (2005), "Glazer faces NFL grilling over conflicts posed by United deal", The Independent, 22/05/05.

HANNERZ U. (1996), Transnational Connections: Culture, People, Places, London, Routledge.

HOBSBAWM E. and RANGER T. (1983), The Invention of Tradition, Cambridge, University Press.

KING A. (1998), The End of the Terraces: The transformation of English football in the 1990s, London, Leicester University Press.

MELLOR G. (1999), "The Social and Geographical Make-up of Football Crowds in the North-West of England, 1946-1962", The Sports Historian No.19, November 1999.

PORTER C. (2005), Football Ownership, Local Identity and Regulation (Paper presented at the 2005 International Conference of Sport and Urban Development at the Rotterdam World Trade Centre, November 11th, 2005, Rotterdam, Netherlands).

ROBERTSON R. (1992), Globalization: social theory and global culture, London, Sage. 